Article

\title{
An Infinite System of Fractional Order with $p$-Laplacian Operator in a Tempered Sequence Space via Measure of Noncompactness Technique
}

\author{
Ahmed Salem ${ }^{1, * \mathbb{C}}$, Lamya Almaghamsi ${ }^{2}$ and Faris Alzahrani ${ }^{1}[$ \\ 1 Department of Mathematics, Faculty of Science, King Abdulaziz University, P.O. Box 80203, \\ Jeddah 21589, Saudi Arabia; falzahrani1@kau.edu.sa \\ 2 Department of Mathematics, University of Jeddah, Jeddah 41510, Saudi Arabia; LALMAGHAMSI@uj.edu.sa \\ * Correspondence: asaalshreef@kau.edu.sa
}

Citation: Salem, A.; Almaghamsi, L.; Alzahrani, F. An Infinite System of Fractional Order with $p$-Laplacian Operator in a Tempered Sequence Space via Measure of Noncompactness Technique. Fractal Fract. 2021, 5, 182. https://doi.org/ 10.3390 / fractalfract5040182

Academic Editors: Giacomo Ascione, Alessandra Meoli and Enrica Pirozzi

Received: 22 September 2021

Accepted: 19 October 2021

Published: 25 October 2021

Publisher's Note: MDPI stays neutral with regard to jurisdictional claims in published maps and institutional affiliations.

Copyright: (c) 2021 by the authors. Licensee MDPI, Basel, Switzerland. This article is an open access article distributed under the terms and conditions of the Creative Commons Attribution (CC BY) license (https:/ / creativecommons.org/licenses/by/ $4.0 /)$.

\begin{abstract}
In the current study, a new class of an infinite system of two distinct fractional orders with $p$-Laplacian operator is presented. Our mathematical model is introduced with the CaputoKatugampola fractional derivative which is considered a generalization to the Caputo and Hadamard fractional derivatives. In a new sequence space associated with a tempered sequence and the sequence space $c_{0}$ (the space of convergent sequences to zero), a suitable new Hausdorff measure of noncompactness form is provided. This formula is applied to discuss the existence of a solution to our infinite system through applying Darbo's theorem which extends both the classical Banach contraction principle and the Schauder fixed point theorem.
\end{abstract}

Keywords: $p$-Laplacian operator; infinite system; Darbo's fixed point theorem; sequence space; measure of noncompactness

MSC: 34A12; 34A08; 47H10; 47H08

\section{Introduction}

Differential and integral equations take place in the research work of $p$-Laplacian equation with $n$-dimensional space, a gas turbulent flow in porous media and non-newtonian fluid (see [1-3] and references cited therein). Infinite systems of fractional differential equations play a considerable role in several nonlinear analysis branches. These systems demonstrate some examples that cover the theorems for neural nets, dissociation of polymers and branching process. Therefore, the study of infinite systems drew the attention of a number of contributors (see [4-8] and references given therein).

It is well known that the concept of diffusion is associated with random motion of particles in space, usually denoted as Brownian motion [9]. Random fractional differential equations are useful mathematical tools to model problems involving memory effects and uncertainties. Since integer order differential equations cannot precisely describe the experimental and field measurement data, as an alternative approach, fractional order differential equation models are now being widely applied [10]. Mainradi and Pironi [9] have revisited the Brownian motion on the basis of the fractional Langevin equation. The two fluctuation-dissipation theorems and of the techniques of the Fractional Calculus have provided the analytical expressions of the correlation functions. The random force has been shown to be represented by a superposition of the usual white noise with a fractional noise. The fractional Langevin differential equation has been under consideration by several contributions [11-14]. Various types of this equation have been proposed and investigated using different approaches, see [15] and the references cited therein. Zhou et al. [15] considered the fractional Langevin differential equation subject to $p$ Laplacian operator in the Caputo sense of the form 


$$
{ }^{c} D^{\mu} \Phi_{p}\left[\left({ }^{c} D^{v}+\lambda\right) u(t)\right]=f\left(t, u(t),{ }^{c} D^{\alpha} u(t)\right), \quad t \in[0,1] .
$$

In spite of the great significance of fractional Langevin equation with $\lambda=0$ (the dissipative parameter), infinite systems and $p$-Laplacian operator in differential equations theory, there is no contributor, as far as we know, that has touched on bringing them together. This is what stimulated us in this paper to present the following system

$$
{ }_{c}^{\rho_{n}} D^{\mu_{n}} \Phi_{p}\left({ }_{c}^{\rho_{n}} D^{v_{n}} u_{n}(t)\right)=f_{n}\left(t, u(t),{ }_{c}^{\rho} D^{\alpha} u(t)\right), \quad t \in[0,1], n \in \mathbb{N}
$$

where $\Phi_{p}(r)=|r|^{p-2} r, p>1$ is the $p$-Laplacian operator, $u(t)=\left(u_{n}(t)\right)$ is a convergent sequence, $f_{n}:[0,1] \times c_{0}^{\beta} \times c_{0}^{\beta} \rightarrow c_{0}^{\beta}$ are continuously differentiable functions, $0<\rho_{n} \leq 1$, $0<\mu_{n} \leq 1,1<v_{n} \leq 2, \alpha=\left(\alpha_{n}\right), 0<\alpha_{n}<v_{n},{ }_{c}^{\rho} D^{\alpha}$ is the Caputo generalized fractional derivative due to Katugampola [16] and the space $c_{0}^{\beta}$ is the tempered space with the tempering sequence $\beta=\left(\beta_{n}\right)$. This system subjects to the assumptions

$$
u_{n}(0)=0, \quad \quad_{c}^{\rho_{n}} D^{\mu_{n}} u_{n}(0)=0, \quad \lim _{t \rightarrow 0} t^{1-\rho_{n}} u_{n}^{\prime}(t)=0 .
$$

The value of the Caputo-Katugampola fractional derivative is found in the verity that it is a generalization of Hadamard and Caputo fractional approaches. It has drawn the attention of many authors who have construct their mathematical models based on it. For more details and properties, see [17]. Zeng et al. [18] provided a numerical method for solving generalized fractional differential equation of the Caputo-Katugampola derivative. Almeida et al. [17] proved an existence and uniqueness solution for a fractional Cauchytype problem and then presented a simple numerical procedure to obtain a decomposition formula for the Caputo-Katugampola derivative. For more contributions, see $[19,20]$ and references given therein.

Unquestionably, the fractional differential equation is a powerful mathematical case for presenting extra flexibility in treatment with numerous real-world implementations. More speciality, the fractional differential equations are extensively used in modeling different phenomena such as diffusion modeling [21], robot manipulators [22], economics [23], and many more. In view of its distinguished interest, several contributors have paid their attentions to deeply discuss the boundary value problems presented based on such equations (see [24-26]).

The measures of noncompactness theory make up an extremely remarkable branch of the nonlinear functional analysis. It permits us to choose an extremely important class of operators as generalizations of compact operators. Those operators satisfy the Darbo contractions with respect to a measure of noncompactness. The measures of noncompactness are widely used in fixed point theory. Darbo fixed point theorem is a good way to investigate the existence and uniqueness of solution to differential and integral equations via applying measures of noncompactness techniques [27,28].

The main goal of this article can be outlined as follows. In a tempered sequence space associated with the classical sequence space $c_{0}$, a suitable Hausdorff measure on noncompactness is presented and used to investigate the infinite system (1) and (2) by aiding of Darbo fixed point theorem which extends both the classical Banach contraction principle and the Schauder fixed point theorem [29].

\section{Preliminaries}

This section is separated into two subsections. The first subsection inserts the main results and basic concepts needed in fractional calculus. The other subsection provides a short survey for the measure of noncompactness.

\subsection{Fractional Calculus}

Katugampola [30] defined a approach of fractional integral as 


$$
\rho^{\mu} f(t)=\frac{\rho^{1-\mu}}{\Gamma(\mu)} \int_{0}^{t} \frac{s^{\rho-1} f(s)}{\left(t^{\rho}-s^{\rho}\right)^{1-\mu}} d s
$$

where $0<\rho \leq 1$ and $\mu>0$, provided that the integral exists. He has shown that it satisfies the semigroup property

$$
{ }^{\rho} I^{v \rho} I^{\mu} f(t)=\rho^{\rho} I^{v+\mu} f(t), \quad v>0, \mu>0 .
$$

It is clear that

$$
{ }^{\rho} I^{\mu} t^{\rho \lambda}=\frac{\Gamma(\lambda+1)}{\rho^{\mu} \Gamma(\mu+\lambda+1)} t^{\rho(\lambda+\mu)}, \quad \mu>0, \lambda>-1 .
$$

Jarad et al. [31] evolved a Katugampola fractional derivative in the Caputo sense [32] to be

$$
\begin{aligned}
{ }_{c}^{\rho} \mathcal{D}^{\mu} f(t) & =\left(\rho^{\rho} I^{n-\mu}\left(t^{1-\rho} \frac{d}{d t}\right)^{n} f\right)(t), \quad n-1<\mu \leq n, n \in \mathbb{N} \\
& =\frac{\rho^{\mu-n+1}}{\Gamma(n-\mu)} \int_{0}^{t} \frac{s^{\rho-1}}{\left(t^{\rho}-s^{\rho}\right)^{\mu-n+1}}\left(s^{1-\rho} \frac{d}{d s}\right)^{n} f(s) d s .
\end{aligned}
$$

It is remarkable to note that the approach above leads to a Caputo-Hadamard derivative when $\rho \rightarrow 0$ and Caputo derivative when $\rho \rightarrow 1$. Some of its properties are provided as [31]

Lemma 1. Suppose $n \in \mathbb{N}, n-1<\mu \leq n$ and $0<\rho \leq 1$. Then, we have

$$
\begin{aligned}
& { }_{c}^{\rho} \mathcal{D}^{\mu} t^{\rho \lambda}=\frac{\rho^{\mu} \Gamma(\lambda+1)}{\Gamma(\lambda-\mu+1)} t^{\rho(\lambda-\mu)}, \quad \lambda>-1, \lambda \neq 0,1, \cdots, n-1 \\
& { }_{c}^{\rho} \mathcal{D}^{\mu} t^{\rho \lambda}=0, \quad \lambda=0,1, \cdots, n-1 \\
& { }_{c}^{\rho} \mathcal{D}^{\mu} A=0, \quad A \text { is a constant } \\
& { }_{c}^{\rho} \mathcal{D}^{\mu \rho} I^{v} f(t)={ }^{\nu} I^{\nu-\mu} f(t), \quad v \geq \mu \\
& \rho^{\mu} I^{\mu}{ }_{c}^{\rho} \mathcal{D}^{\mu} f(t)=f(t)-\sum_{r=0}^{n-1} a_{r} t^{\rho r}, \quad a_{r}, r=0,1, \cdots, n-1, \text { are constants. }
\end{aligned}
$$

Lemma 2. The $p$-Laplacian operator $\Phi_{p}, p>1$ satisfies

(1) $\Phi_{p}$ is increasing, continuous and invertible with inverse $\Phi_{p}^{-1}=\Phi_{q}$ where $q>1$ and $1 / p+1 / q=1$.

(2) $\left|\Phi_{p}(x)\right|=\Phi_{p}(|x|)$ and $\Phi_{p}(\lambda x)=\lambda^{p-1} \Phi_{p}(x), \lambda>0$

(3) Ref. [3] For all $x, y>0$

$$
\begin{aligned}
& \Phi_{p}(x+y) \leq \Phi_{p}(x)+\Phi_{p}(y), \quad \text { if } p<2 \\
& \Phi_{p}(x+y) \leq 2^{p-2}\left(\Phi_{p}(x)+\Phi_{p}(y)\right), \quad \text { if } p \geq 2 .
\end{aligned}
$$

(4) Refs. [33,34] For all $x, y \in \mathbb{R}$, there exist $c_{1}>0$ and $c_{2}>0$ satisfy

$$
\begin{array}{ll}
\left|\Phi_{p}(x)-\Phi_{p}(y)\right| \leq c_{1}|x-y|^{p-1} & \text { if } \quad 1<p \leq 2 \\
\left|\Phi_{p}(x)-\Phi_{p}(y)\right| \leq c_{2}(|x|+|y|)^{p-2}|x-y| & \text { if } \quad p>2 .
\end{array}
$$

Lemma 3. Suppose that the function $f:[0,1] \rightarrow \mathbb{R}$ is a continuous function, $\rho \in(0,1], \mu \in(0,1]$ and $v \in(1,2]$. Then, the boundary value problem

$$
{ }_{c}^{\rho} D^{\mu} \Phi_{p}\left({ }_{c}^{\rho} D^{v} u(t)\right)=f(t), \quad t \in[0,1], p \geq 1
$$


subject to the assumptions

$$
u(0)=0, \quad \rho_{c}^{\rho} D^{v} u(0)=0, \quad \lim _{t \rightarrow 0} t^{1-\rho} u^{\prime}(t)=0
$$

has the unique solution

$$
u(t)=\frac{\rho^{1-v}}{\Gamma(v)} \int_{0}^{t} \frac{s^{\rho-1}}{\left(t^{\rho}-s^{\rho}\right)^{1-v}} \Phi_{q}\left(\frac{\rho^{1-\mu}}{\Gamma(\mu)} \int_{0}^{s} \frac{r^{\rho-1} f(r)}{\left(s^{\rho}-r^{\rho}\right)^{1-\mu}} d r\right) d s
$$

Proof. Operate by ${ }^{\rho} I^{\mu}$ on both sides of (7) with using the last statement of Lemma 1 to obtain

$$
\Phi_{p}\left({ }_{c}^{\rho} D^{v} u(t)\right)={ }^{\rho} I^{\mu} f(t)+a_{0} .
$$

Taking the $p$-Laplacian inverse and the second condition in (8) produces

$$
{ }_{c}^{\rho} D^{v} u(t)=\Phi_{q}\left(\rho^{\mu} I^{\mu} f(t)\right) .
$$

Again, operating by ${ }^{\rho} I^{v}$ on all sides of the former equation and inserting the last item of Lemma 1 obtains

$$
u(t)={ }^{\rho} I^{v} \Phi_{q}\left({ }^{\rho} I^{\mu} f(t)\right)+a_{1}+a_{2} t^{\rho} .
$$

Inserting the first and last conditions produces $a_{1}=a_{2}=0$. By the definition (3), one can obtain (3). Conversely, by substituting (9) into the left side in (7) and applying Lemma 1, we can obtain the right side of Equation (9). It is easy to view that the solution (9) verifies all assumptions of (8).

\subsection{Hausdorff Measure of Noncompactness and Tempered Sequence Space}

Let $c_{0}$ be the sequence space of all sequences $u=\left(u_{n}\right)$ converging to zero and $\beta=\left(\beta_{n}\right)$ be a positive non-increasing real sequence. Such a sequence is called the tempering sequence. Banas and Krajewska [35] have presented the tempered sequence space

$$
c_{0}^{\beta}=\left\{u \in c_{0} \mid \beta_{n} u_{n} \rightarrow 0 \text { as } n \rightarrow \infty\right\}
$$

and proved that the space $c_{0}^{\beta}$ is a Banach space equipped the norm

$$
\|u\|_{c_{0}^{\beta}}=\sup _{n \in \mathbb{N}}\left\{\beta_{n}\left|u_{n}\right|, u \in c_{0}\right\} .
$$

It is worth pointing that if the tempering sequence $\beta$ is a constant sequence or it is bounded from below, then the norm in the tempered sequence space $c_{0}^{\beta}$ is equivalent to the classical supremum norm in $c_{0}$. They have proved that the Hausdorff measure of noncompactness can be given by the formula

$$
\chi_{c_{0}^{\beta}}(\mathscr{B})=\lim _{m \rightarrow \infty} \sup _{n \geq m}\left\{\beta_{n}\left|u_{n}\right|, u \in \mathscr{B}\right\}
$$

where $\mathscr{B}$ is subset of a nonempty bounded set of the Banach space $c_{0}^{\beta}$ and the Hausdorff measure of noncompactness is the mapping $\chi: \mathscr{B} \rightarrow[0, \infty)$ defined by

$$
\chi(\mathscr{B})=\inf \{\epsilon>0 \mid \mathscr{B} \text { has a finite } \epsilon-\text { net in Banach space }\} .
$$

For extra details of Hausdorff measure of noncompactness, see [29,36]. 
Theorem 1 (Darbo Theorem [29]). Suppose $\mathscr{B}$ is a closed, bounded, convex and nonempty subset of a Banach space $X$. Let $F: \mathscr{B} \rightarrow \mathscr{B}$ be a continuous map and there exists a positive constant $k \in[0,1)$ satisfies the property $\chi(F \mathscr{B}) \leq k \chi(\mathscr{B})$. Then, $F$ has a fixed point in $\mathscr{B}$.

\section{Basic Constructions and Main Results}

Consider $\mathcal{C}\left([0,1], c_{0}^{\beta}\right)$ is the space contains continuous sequence functions defined on the interval $[0,1]$ and belong to the space $c_{0}^{\beta}$ : That is $u(t) \in \mathcal{C}\left([0,1], c_{0}^{\beta}\right)$ leads to $\beta_{n} u_{n}(t) \rightarrow 0$ as $n \rightarrow \infty$ and $u_{n}:[0,1] \rightarrow \mathbb{R}$ is continuous function for all $n \in \mathbb{N}$ where $\beta=\left(\beta_{n}\right)$ is a positive non-increasing sequence with $\beta_{\infty} \neq 0$. Consider the space

$$
\mathbb{X}=\left\{u(t) \mid u \in \mathcal{C}\left([0,1], c_{0}^{\beta}\right) \text { and }{ }_{c}^{\rho} D^{\alpha} u(t) \in \mathcal{C}\left([0,1], c_{0}^{\beta}\right)\right\}
$$

where $0<\alpha<v$ and $1<v \leq 2$. It is easy to see that the space $\mathbb{X}$ is Banach space under the norm

$$
\|u\|=\|u\|_{c_{0}^{\beta}}+\left\|{ }_{c}^{\rho} D^{\alpha} u\right\|_{c_{0}^{\beta}} .
$$

Based on to the Formula (10) and Theorem 1.3 in [6], we can derive the Hausdorff measure of noncompactness in the form

$$
\chi_{\mathbb{X}}(\mathscr{B})=\lim _{m \rightarrow \infty} \sup _{n \geq m}\left\{\max _{t \in[0,1]}\left\{\beta_{n}\left|u_{n}(t)\right|\right\}+\max _{t \in[0,1]}\left\{\left.\beta_{n}\right|_{c} ^{\rho_{n}} D^{\alpha_{n}} u_{n}(t) \mid\right\}, u \in \mathscr{B}\right\}
$$

where $\mathscr{B}$ is subset of a nonempty bounded set of the Banach space $\mathbb{X}$.

It is clear that the unique solution of the infinite system (1) and (2) comes immediately by replacing $u, \mu, v, \alpha, \rho$ and $f$ by $u_{n}, \mu_{n}, v_{n}, \alpha_{n}, \rho_{n}$ and $f_{n}$, respectively, in Lemma 3. Our discussion of the existence results for the infinite system (1) and (2) will be investigated under the following assumptions:

$\left(\mathcal{H}_{1}\right)$ The functions $f_{n}:[0,1] \times c_{0}^{\beta} \times c_{0}^{\beta} \rightarrow c_{0}^{\beta}$ are continuous for all $n \in \mathbb{N}$ and satisfy the Lipschitz condition with Lipschitz constant $L$ as

$$
\left|f_{n}\left(t, u_{1}, v_{1}\right)-f_{n}\left(t, u_{2}, v_{2}\right)\right| \leq L\left(\left|u_{1}-u_{2}\right|+\left|v_{1}-v_{2}\right|\right), \quad u_{i}, v_{i} \in c_{0}^{\beta}, i=1,2 .
$$

$\left(\mathcal{H}_{2}\right)$ There exist nonnegative sequence functions, $g_{n}(t)$ and $h_{n}(t)$, that satisfy the inequality

$$
\left|f_{n}(t, u, v)\right| \leq g_{n}(t)+h_{n}(t)\left(\left|u_{n}(t)\right|^{p-1}+\left|v_{n}(t)\right|^{p-1}\right)
$$

for all $n \in \mathbb{N}, p>1, t \in[0,1]$ and $u, v \in \mathcal{C}\left([0,1], c_{0}^{\beta}\right)$.

$\left(\mathcal{H}_{3}\right)$ There are positive constants $\mathrm{A}$ and $\mathrm{C}$ such that

$$
\mathrm{A}=\sup _{n \in \mathbb{N}} \max _{t \in[0,1]} \beta_{n}\left|g_{n}(t)\right|^{q-1} \quad \text { and } \quad \mathrm{C}=\sup _{n \in \mathbb{N}} \max _{t \in[0,1]}\left|h_{n}(t)\right|^{q-1}, \quad q>1 .
$$

For the convenance of computations, let

$$
\Delta_{v}=\sup _{n \in \mathbb{N}} \frac{\rho_{n}^{\mu_{n}(1-q)-v_{n}} \Gamma\left(\mu_{n}(q-1)+1\right)}{\Gamma^{q-1}\left(\mu_{n}+1\right) \Gamma\left(\mu_{n}(q-1)+v_{n}+1\right)} .
$$

Theorem 2. Under the suppositions above, the infinite system (1) and (2) has at least one solution in $\mathbb{X}$ for all $p>1$ provided that $\Delta<1$ where

$$
\Delta=4^{(q-2) H(q-2)} \mathrm{C}\left(\Delta_{v}+\Delta_{v-\alpha}\right), \quad \frac{1}{p}+\frac{1}{q}=1
$$

and $H(\cdot)$ is the Heaviside step function. 
Proof. Define the operator $\mathbf{F}_{p}: \mathbb{X} \rightarrow \mathbb{X}$ such that

$$
\left(\mathbf{F}_{p} u_{n}\right)(t)={ }^{\rho_{n}} I^{v_{n}} \Phi_{q}\left({ }^{\rho_{n}} I^{\mu_{n}} f_{n}\left(t, u(t),{ }_{c}^{\rho} D^{\alpha} u(t)\right)\right) .
$$

By the continuity of $f_{n}, n \in \mathbb{N}$ according to $\left(\mathcal{H}_{1}\right)$, the operator $\mathbf{F}_{p}$ is continuous for all $p>1$. Furthermore, it is not difficult with using Lemmas 1 and 2 to show

$$
\begin{aligned}
& \left|\left(\mathbf{F}_{p} u_{n}\right)(t)\right|=\left|\rho^{\rho_{n}} I^{v_{n}} \Phi_{q}\left({ }^{\rho_{n}} I^{\mu_{n}} f_{n}\left(t, u(t),{ }_{c}^{\rho} D^{\alpha} u(t)\right)\right)\right| \leq{ }^{\rho_{n}} I^{v_{n}} \Phi_{q}\left(\rho^{\rho_{n}} I^{\mu_{n}}\left|f_{n}\left(t, u(t),{ }_{c}^{\rho} D^{\alpha} u(t)\right)\right|\right) \\
& \text { In view of }\left(\mathcal{H}_{2}\right) \text { and }\left(\mathcal{H}_{3}\right) \text { with using the the relation (5) and the facts } \\
& \Phi_{q}(x y)=\Phi_{q}(x) \Phi_{q}(y) \text { and } \Phi_{q}(x)=x^{q-1}, x>0 \text {, we find that } \\
& \begin{array}{l}
\beta_{n} \Phi_{q}\left(\rho_{n} I^{\mu_{n}}\left|f_{n}\left(t, u(t),{ }_{c}^{\rho} D^{\alpha} u(t)\right)\right|\right) \\
\leq \beta_{n} \Phi_{q}\left(\rho_{n} I^{\mu_{n}}\left[g_{n}(t)+\left.h_{n}(t)\left(\left|u_{n}(t)\right|^{p-1}+\mid{ }_{c}^{\rho} D^{\alpha} u(t)\right)\right|^{p-1}\right]\right) \\
\left.=\Phi_{q}\left(\rho_{n} I^{\mu_{n}}\left[\beta_{n}^{p-1} g_{n}(t)+h_{n}(t)\left(\left(\beta_{n}\left|u_{n}(t)\right|\right)^{p-1}+\left(\beta_{n} \mid{ }_{c}^{\rho} D^{\alpha} u(r)\right) \mid\right)^{p-1}\right)\right]\right) \\
\left.=\Phi_{q}\left(\mathrm{~A}^{p-1}+\mathrm{C}^{p-1}\left(\|u\|_{c_{0}^{\beta}}^{p-1}+\|{ }_{c}^{\rho} D^{\alpha} u\right) \|_{c_{0}^{\beta}}^{p-1}\right)\right) \Phi_{q}\left({ }_{n}^{\rho_{n}} I^{\mu_{n}} 1\right) \\
\left.=\Phi_{q}\left(\mathrm{~A}^{p-1}+\mathrm{C}^{p-1}\left(\|u\|_{c_{0}^{\beta}}^{p-1}+\|{ }_{c}^{\rho} D^{\alpha} u\right) \|_{c_{0}^{\beta}}^{p-1}\right)\right) \Phi_{q}\left(\frac{{ }^{\rho_{n} \mu_{n}}}{\rho_{n}^{\mu_{n}} \Gamma\left(\mu_{n}+1\right)}\right) \\
\left.=\frac{{ }^{\rho_{n} \mu_{n}(q-1)}}{\rho_{n}^{\mu_{n}(q-1)} \Gamma^{q-1}\left(\mu_{n}+1\right)} \Phi_{q}\left(\mathrm{~A}^{p-1}+\mathrm{C}^{p-1}\left(\|u\|_{c_{0}^{\beta}}^{p-1}+\|{ }_{c}^{\rho} D^{\alpha} u\right) \|_{c_{0}^{\beta}}^{p-1}\right)\right)
\end{array}
\end{aligned}
$$

From the third statement of Lemma 2, we have

$$
\left.\Phi_{q}\left(\mathrm{~A}^{p-1}+\mathrm{C}^{p-1}\left(\|u\|_{c_{0}^{\beta}}^{p-1}+\|{ }_{c}^{\rho} D^{\alpha} u\right) \|_{c_{0}^{\beta}}^{p-1}\right)\right) \leq 2^{(q-2) H(q-2)} \mathrm{A}+4^{(q-2) H(q-2)} \mathrm{C}\|u\|
$$

Hence, we obtain

$$
\begin{aligned}
& \left\|\mathbf{F}_{p} u\right\|_{c_{0}^{\beta}}=\sup _{n \in \mathbb{N}} \max _{t \in[0,1]} \beta_{n}\left|\left(\mathbf{F}_{p} u_{n}\right)(t)\right| \\
& =\left(2^{(q-2) H(q-2)} \mathrm{A}+4^{(q-2) H(q-2)} \mathrm{C}\|u\|\right) \sup _{n \in \mathbb{N}} \max _{t \in[0,1]} \frac{\rho_{n}^{\mu_{n}(1-q)}}{\Gamma^{q-1}\left(\mu_{n}+1\right)} \rho_{n} I^{v_{n}} t^{\rho_{n} \mu_{n}(q-1)} \\
& =\left(2^{(q-2) H(q-2)} \mathrm{A}+4^{(q-2) H(q-2)} \mathrm{C}\|u\|\right) \sup _{n \in \mathbb{N}} \max _{t \in[0,1]} \frac{\rho_{n}^{\mu_{n}(1-q)-v_{n}} \Gamma\left(\mu_{n}(q-1)+1\right) \rho^{\rho_{n}\left(\mu_{n}(q-1)+v_{n}\right)}}{\Gamma^{q-1}\left(\mu_{n}+1\right) \Gamma\left(\mu_{n}(q-1)+v_{n}+1\right)} \\
& =\left(2^{(q-2) H(q-2)} \mathrm{A}+4^{(q-2) H(q-2)} \mathrm{C}\|u\|\right) \sup _{n \in \mathbb{N}} \frac{\rho_{n}^{\mu_{n}(1-q)-v_{n}} \Gamma\left(\mu_{n}(q-1)+1\right)}{\Gamma^{q-1}\left(\mu_{n}+1\right) \Gamma\left(\mu_{n}(q-1)+v_{n}+1\right)} \\
& =\left(2^{(q-2) H(q-2)} \mathrm{A}+4^{(q-2) H(q-2)} \mathrm{C}\|u\|\right) \Delta_{v}
\end{aligned}
$$

where $\Delta_{v}$ is defined in (13). From the $4^{\text {th }}$ statement of Lemma 1, we have

$$
\left({ }_{c}^{\rho_{n}} D^{\alpha_{n}} \mathbf{F}_{p} u_{n}\right)(t)=\rho_{n} I^{v_{n}-\alpha_{n}} \Phi_{q}\left({ }^{\rho_{n}} I^{\mu_{n}}\left|f_{n}\left(t, u(t),{ }_{c}^{\rho} D^{\alpha} u(t)\right)\right|\right)
$$

Similarly, we have

$$
\left\|{ }_{c}^{\rho} D^{\alpha} \mathbf{F}_{p} u\right\|_{c_{0}^{\beta}} \leq\left(2^{(q-2) H(q-2)} \mathrm{A}+4^{(q-2) H(q-2)} \mathrm{C}\|u\|\right) \Delta_{v-\alpha}
$$


These conclude that

$$
\left\|\mathbf{F}_{p} u\right\|=\left\|\mathbf{F}_{p} u\right\|_{c_{0}^{\beta}}+\left\|{ }_{c}^{\rho} D^{\alpha} \mathbf{F}_{p} u\right\|_{c_{0}^{\beta}} \leq\left(2^{(q-2) H(q-2)} \mathrm{A}+4^{(q-2) H(q-2)} C\|u\|\right)\left(\Delta_{v}+\Delta_{v-\alpha}\right)
$$

which leads to the boundedness of the operator $\mathbf{F}_{p} u$ for all $p>1$. Now, we present the set $\mathscr{B} \subset \mathbb{X}$ such that

$$
\mathscr{B}=\{u \in \mathbb{X} \mid\|u\| \leq r, u \text { holds the conditions (2) }\}
$$

The subspace $\mathscr{B}$ is bounded, closed and convex, with fix radius $r$ satisfies

$$
\left(2^{(q-2) H(q-2)} \mathrm{A}+4^{(q-2) H(q-2)} \mathrm{C} r\right)\left(\Delta_{v}+\Delta_{v-\alpha}\right) \leq r .
$$

It is obvious that the operator $\mathbf{F}_{p}: \mathscr{B} \rightarrow \mathscr{B}$ is bounded. In order to prove the continuity of $\mathbf{F}_{p}$ on the set $\mathscr{B}$, let $u, v \in \mathscr{B}$ and for all $\epsilon>0$ there exists

$$
\begin{aligned}
& 0<\delta<\max \left\{\frac{\epsilon^{p-1} \beta_{1}^{2-p}}{L c_{1}^{p-1} \Delta_{v}^{p-1}}, \frac{\epsilon^{p-1} \beta_{1}^{2-p}}{L c_{1}^{p-1} \Delta_{v-\alpha}^{p-1}}\right\}, \quad 1<q \leq 2, p>2 \\
& 0<\delta<\max \left\{\frac{\epsilon \beta_{\infty}^{2-p}\left(\mathrm{~A}+4^{q-2} \mathrm{C} r\right)^{p-2}}{2^{q-1} L c_{2} \Delta_{v}}, \frac{\epsilon \beta_{\infty}^{2-p}\left(\mathrm{~A}+4^{q-2} \mathrm{C} r\right)^{p-2}}{2^{q-1} L c_{2} \Delta_{v-\alpha}}\right\}, \quad 1<p \leq 2, q>2
\end{aligned}
$$

where $L$ is Lipschitz constant $\left(\mathcal{H}_{1}\right)$ such that $\|u-v\|<\delta$. In fact, we obtain

$$
\left|\left(\mathbf{F}_{p} u_{n}\right)(t)-\left(\mathbf{F}_{p} v_{n}\right)(t)\right| \leq \rho_{n} I^{v_{n}}\left|\Phi_{q}\left(\rho_{n} I^{\mu_{n}} f_{n}\left(t, u(t),{ }_{c}^{\rho} D^{\alpha} u(t)\right)\right)-\Phi_{q}\left(\rho_{n} I^{\mu_{n}} f_{n}\left(t, v(t),{ }_{c}^{\rho} D^{\alpha} v(t)\right)\right)\right|
$$

When $1<q \leq 2$, using the $4^{\text {th }}$ statement in Lemma 2 and $\left(\mathcal{H}_{1}\right)$ obtains

$$
\begin{aligned}
& \left\|\mathbf{F}_{p} u-\mathbf{F}_{p} v\right\|_{c_{0}^{\beta}}=\sup _{n \in \mathbb{N}} \max _{t \in[0,1]} \beta_{n}\left|\left(\mathbf{F}_{p} u_{n}\right)(t)-\left(\mathbf{F}_{p} v_{n}\right)(t)\right| \\
& \leq c_{1} \sup _{n \in \mathbb{N}} \max _{t \in[0,1]} \beta_{n}^{2-q} \rho_{n} I^{v_{n}}\left(\rho_{n} I^{\mu_{n}} \beta_{n}\left|f_{n}\left(t, u(t),{ }_{c}^{\rho} D^{\alpha} u(t)\right)-f_{n}\left(t, v(t),{ }_{c}^{\rho} D^{\alpha} v(t)\right)\right|\right)^{q-1} \\
& \leq c_{1} L^{q-1} \sup _{n \in \mathbb{N}} \max _{t \in[0,1]} \beta_{n}^{2-q} \rho_{n} I^{v_{n}}\left(\rho_{n} I^{\mu_{n}}\left(\beta_{n}|u(t)-v(t)|+\beta_{n} \mid{ }_{c}^{\rho} D^{\alpha} u(t)-{ }_{c}^{\rho} D^{\alpha} v(t)\right) \mid\right)^{q-1} \\
& \leq c_{1} L^{q-1} \sup _{n \in \mathbb{N}} \max _{t \in[0,1]} \beta_{n}^{2-q} \rho_{n} I^{v_{n}}\left(\rho_{n} I^{\mu_{n}} 1\right)^{q-1}\|u-v\|^{q-1} \\
& \leq c_{1} L^{q-1} \beta_{1}^{2-q} \Delta_{n} u\|u-v\|^{q-1} \leq c_{1} L^{q-1} \beta_{1}^{2-q} \Delta_{v} \delta^{q-1}<\frac{\epsilon}{2}
\end{aligned}
$$

and

$$
\left\|{ }_{c}^{\rho} D^{\alpha} \mathbf{F}_{p} u-{ }_{c}^{\rho} D^{\alpha} \mathbf{F}_{p} v\right\|_{c_{0}^{\beta}} \leq c_{1} L^{q-1} \beta_{1}^{2-q} \Delta_{n} u\|u-v\|^{q-1} \leq c_{1} L^{q-1} \beta_{1}^{2-q} \Delta_{v-\alpha} \delta^{q-1}<\frac{\epsilon}{2}
$$

which mean that $\left\|\mathbf{F}_{p} u-\mathbf{F}_{p} v\right\| \leq \epsilon$ and so the operator $\mathbf{F}_{p}$ is continuous on the set $\mathscr{B}$ when $1<q \leq 2$. Similarly, when $q>2$, we find that

$$
\begin{aligned}
\left\|\mathbf{F}_{p} u-\mathbf{F}_{p} v\right\|_{c_{0}^{\beta}} & \leq c_{2} \sup _{n \in \mathbb{N}} \max _{t \in[0,1]} \rho_{n} I^{v_{n}}\left[{ }^{\rho_{n}} I^{\mu_{n}} \beta_{n}\left|f_{n}\left(t, u(t),{ }_{c}^{\rho} D^{\alpha} u(t)\right)-f_{n}\left(t, v(t),{ }_{c}^{\rho} D^{\alpha} v(t)\right)\right|\right. \\
& \left.\times\left({ }^{\rho_{n}} I^{\mu_{n}}\left[\left|f_{n}\left(t, u(t),{ }_{c}^{\rho} D^{\alpha} u(t)\right)\right|+\left|f_{n}\left(t, v(t),{ }_{c}^{\rho} D^{\alpha} v(t)\right)\right|\right]\right)^{q-2}\right] .
\end{aligned}
$$

It is easy to see that

$$
{ }^{\rho_{n}} I^{\mu_{n}} \beta_{n}\left|f_{n}\left(t, u(t),{ }_{c}^{\rho} D^{\alpha} u(t)\right)-f_{n}\left(t, v(t),{ }_{c}^{\rho} D^{\alpha} v(t)\right)\right| \leq \frac{L t^{\rho_{n} \mu_{n}}}{\rho_{n}^{\mu_{n}} \Gamma\left(\mu_{n}+1\right)}\|u-v\|
$$


and, with noting $q-2=(2-p)(q-1)$,

$$
\begin{aligned}
& \left({ }^{\rho_{n}} I^{\mu_{n}}\left[\left|f_{n}\left(t, u(t),{ }_{c}^{\rho} D^{\alpha} u(t)\right)\right|+\left|f_{n}\left(t, v(t),{ }_{c}^{\rho} D^{\alpha} v(t)\right)\right|\right]\right)^{q-2} \\
& \leq\left({ }^{\rho_{n}} I^{\mu_{n}}\left[2 g_{n}(t)+h_{n}(t)\left(|u|^{p-1}+\left|{ }_{c}^{\rho} D^{\alpha} u(t)\right|^{p-1}+|v|^{p-1}+\left|{ }_{c}^{\rho} D^{\alpha} v(t)\right|^{p-1}\right)\right]\right)^{(2-p)(q-1)} \\
& \leq \beta_{n}^{p-2}\left(2^{q-1} \mathrm{~A}+2^{3 q-6} \mathrm{C}(\|u\|+\|v\|)\right)^{2-p}\left({ }^{\rho_{n}} I^{\mu_{n}} 1\right)^{q-2} \\
& \left.\leq \beta_{n}^{p-2}\left(2^{q-1} \mathrm{~A}+2^{3 q-5} \mathrm{C} r\right)\right)^{2-p}\left(\frac{t^{\rho_{n} \mu_{n}}}{\rho_{n}^{\mu_{n}} \Gamma\left(\mu_{n}+1\right)}\right)^{q-2} \\
& =2^{q-2} \beta_{n}^{p-2}\left(\mathrm{~A}+4^{q-1} \mathrm{C} r\right)^{2-p}\left(\frac{t^{\rho_{n} \mu_{n}}}{\rho_{n}^{\mu_{n}} \Gamma\left(\mu_{n}+1\right)}\right)^{q-2}
\end{aligned}
$$

Hence, for $q>2$ and $1<p<2$, we have

$$
\begin{aligned}
\left\|\mathbf{F}_{p} u-\mathbf{F}_{p} v\right\|_{c_{0}^{\beta}} & \leq 2^{q-2}\left(\mathrm{~A}+4^{q-1} \mathrm{C} r\right)^{2-p} L c_{2} \\
& \times \sup _{n \in \mathbb{N}} \max _{t \in[0,1]} \beta_{n}^{p-2} \rho_{n} I^{v_{n}}\left(\frac{t^{\rho_{n} \mu_{n}(q-1)}}{\rho_{n}^{\mu_{n}(q-1)} \Gamma^{q-1}\left(\mu_{n}+1\right)}\right)\|u-v\| \\
& \leq 2^{q-2} \beta_{\infty}^{p-2}\left(\mathrm{~A}+4^{q-1} \mathrm{C} r\right)^{2-p} L c_{2} \Delta_{v} \delta
\end{aligned}
$$

and

$$
\left\|{ }_{c}^{\rho} D^{\alpha} \mathbf{F}_{p} u-{ }_{c}^{\rho} D^{\alpha} \mathbf{F}_{p} v\right\|_{c_{0}^{\beta}} \leq 2^{q-2} \beta_{\infty}^{p-2}\left(\mathrm{~A}+4^{q-1} \mathrm{C} r\right)^{2-p} L c_{2} \Delta_{v-\alpha} \delta
$$

which mean that $\left\|\mathbf{F}_{p} u-\mathbf{F}_{p} v\right\| \leq \epsilon$ and so the operator $\mathbf{F}_{p}$ is continuous on the set $\mathscr{B}$ when $q>2$. To prove that the operator $\mathbf{F}_{p} u(t)$ is continuous uniformly on the interval $[0,1]$, let $0 \leq t_{1}<t_{2} \leq 1$. Then, we can obtain that

$$
\begin{aligned}
& \left|\mathbf{F}_{p} u\left(t_{2}\right)-\mathbf{F}_{p} u\left(t_{1}\right)\right|=\mid \frac{\rho_{n}^{1-v_{n}}}{\Gamma\left(v_{n}+1\right)} \int_{0}^{t_{2}} \frac{s^{\rho_{n}-1}}{\left(t_{2}^{\rho_{n}}-s^{\rho_{n}}\right)^{1-v_{n}}} \Phi_{q}\left({ }^{\rho_{n}} I^{\mu_{n}} f_{n}\left(s, u(s),{ }_{c}^{\rho} D^{\alpha} u(s)\right)\right) d s \\
& -\frac{\rho_{n}^{1-v_{n}}}{\Gamma\left(v_{n}+1\right)} \int_{0}^{t_{1}} \frac{s^{\rho_{n}-1}}{\left(t_{1}^{\rho_{n}}-s^{\rho_{n}}\right)^{1-v_{n}}} \Phi_{q}\left({ }^{\rho_{n}} I^{\mu_{n}} f_{n}\left(s, u(s),{ }_{c}^{\rho} D^{\alpha} u(s)\right)\right) d s \mid \\
& \leq \frac{\Omega \rho_{n}^{1-v_{n}}}{\Gamma\left(v_{n}+1\right)}\left(\int_{0}^{t_{1}}\left(\frac{s^{\rho_{n}-1}}{\left(t_{2}^{\rho_{n}}-s^{\rho_{n}}\right)^{1-v_{n}}}-\frac{s^{\rho_{n}-1}}{\left(t_{1}^{\rho_{n}}-s^{\rho_{n}}\right)^{1-v_{n}}}\right) d s+\int_{t_{1}}^{t_{2}} \frac{s^{\rho_{n}-1}}{\left(t_{2}^{\rho_{n}}-s^{\rho_{n}}\right)^{1-v_{n}}} d s\right) \\
& \leq \frac{\Omega \rho_{n}^{1-v_{n}}}{\Gamma\left(v_{n}+1\right)}\left(\int_{0}^{t_{2}} \frac{s^{\rho_{n}-1}}{\left(t_{2}^{\rho_{n}}-s^{\rho_{n}}\right)^{1-v_{n}}} d s-\int_{0}^{t_{1}} \frac{s^{\rho_{n}-1}}{\left(t_{1}^{\rho_{n}}-s^{\rho_{n}}\right)^{1-v_{n}}} d s\right. \\
& +\int_{0}^{t_{2}} \frac{s^{\rho_{n}-1}}{\left.\left(t_{2}^{\rho_{n}}-s^{\rho_{n}}\right)^{1-v_{n}} d s-\int_{0}^{t_{1}} \frac{s^{\rho_{n}-1}}{\left(t_{2}^{\rho_{n}}-s^{\rho_{n}}\right)^{1-v_{n}}} d s\right)} \\
& \leq \frac{2 \Omega \rho_{n}^{1-v_{n}}}{\Gamma\left(v_{n}+1\right)}\left(\int_{0}^{t_{2}} \frac{s^{\rho_{n}-1}}{\left(t_{2}^{\rho_{n}}-s^{\rho_{n}}\right)^{1-v_{n}}} d s-\int_{0}^{t_{1}} \frac{s^{\rho_{n}-1}}{\left(t_{1}^{\rho_{n}}-s^{\rho_{n}}\right)^{1-v_{n}}} d s\right) \\
& =\frac{2 \Omega}{\rho_{n}^{v_{n}} \Gamma\left(v_{n}+1\right)}\left(t_{2}^{\rho_{n} v_{n}}-t_{1}^{\rho_{n} v_{n}}\right)
\end{aligned}
$$

where $\Omega=\Phi\left(\left|f_{n}\right|\right)$, which tends to zero uniformly as $t_{1} \rightarrow t_{2}$. Similarly, we have

$$
\left|{ }_{c}^{\rho} D^{\alpha} \mathbf{F}_{p} u\left(t_{2}\right)-{ }_{c}^{\rho} D^{\alpha} \mathbf{F}_{p} u\left(t_{1}\right)\right|=\frac{2 \Omega}{\rho_{n}^{v_{n}-\alpha_{n}} \Gamma\left(v_{n}-\alpha_{n}+1\right)}\left(t_{2}^{\rho_{n}\left(v_{n}-\alpha_{n}\right)}-t_{1}^{\rho_{n}\left(v_{n}-\alpha_{n}\right)}\right) .
$$


Thus, the operator $\mathbf{F}_{p} u(t)$ is continuous uniformly on $[0,1]$. The measure of noncompactness due to Hausdorff is evaluated, as above, by using (12) as

$$
\begin{aligned}
\chi_{\mathbb{X}}(\mathbf{F} \mathscr{B}) & =\lim _{m \rightarrow \infty} \sup _{n \geq m}\left\{\max _{t \in[0,1]}\left\{\beta_{n}\left|\mathbf{F}_{p} u_{n}(t)\right|\right\}+\max _{t \in[0,1]}\left\{\beta_{n}||_{c}^{\rho_{n}} D^{\alpha_{n}} \mathbf{F}_{p} u_{n}(t) \mid\right\}, u \in \mathscr{B}\right\} \\
& \leq 4^{(q-2) H(q-2)} \mathrm{C}\left(\Delta_{v}+\Delta_{v-\alpha}\right) \lim _{m \rightarrow \infty} \sup _{n \geq m} \max _{t \in[0,1]} \beta_{n}\left(\left|u_{n}(t)\right|+\left|{ }_{c}^{\rho_{n}} D^{\alpha_{n}} u_{n}(t)\right|\right) \\
& =4^{(q-2) H(q-2)} \mathrm{C}\left(\Delta_{v}+\Delta_{v-\alpha}\right) \chi_{\mathbb{X}}\left(u_{n}(t)\right)=\Delta \chi_{\mathbb{X}}\left(u_{n}(t)\right) .
\end{aligned}
$$

Since $\Delta<1$, according Darbo fixed point theorem, then our system (1)-(2) has at least a solution in the set $\mathscr{B}$ on the unit interval $[0,1]$.

\section{Illustrated Numerical Example}

Let us provide the example below:

$$
{ }_{c}^{\frac{1}{2}} D^{\frac{3}{4}} \Phi_{3}\left({ }_{c}^{\frac{1}{2}} D^{\frac{3}{2}} u_{n}(t)\right)=f_{n}\left(t, u(t), t^{\frac{1}{2}} u^{\prime}(t)\right), \quad t \in[0,1], n \in \mathbb{N} .
$$

This system subject to the assumptions

$$
u_{n}(0)=0, \quad c_{c}^{\frac{1}{2}} D^{\frac{3}{4}} u_{n}(0)=0, \quad \lim _{t \rightarrow 0} t^{\frac{1}{2}} u_{n}^{\prime}(t)=0
$$

$\rho_{n}=1 / 2, \mu_{n}=3 / 4, v_{n}=3 / 2, p=3, q=3 / 2, \alpha=1$. Furthermore, we take the tempered sequence $\left(\beta_{n}\right)=(1+1 / n)$ and

$$
f_{n}(t, y, z)=\frac{e^{-n t} \cos ^{2}(n t)}{(t+1)^{2}}+\frac{t^{2} e^{-n t}}{2(2 \pi-t)^{2}(t+n)^{2}} \sum_{i=n}^{\infty} \frac{\sin ^{2}(i \pi t)}{(i+t)^{2}}\left(y_{i}^{2}+z_{i}^{2}\right) .
$$

It is obvious that the tempered sequence $\left(\beta_{n}\right)=(1+1 / n)$ is a positive decreasing sequence for all $n \in \mathbb{N}$ with $\beta_{\infty}=1 \neq 0$. Furthermore, one can check that $h_{n} \in c_{0}$ and $h_{n} \in c_{0}^{\beta}$. In order to verify the assumption $\left(\mathcal{H}_{1}\right)$. Let $y, y^{\prime}, z, z^{\prime} \in c_{0}^{\beta}$. Then,

$$
\begin{aligned}
\left|f_{n}(t, y, z)-f_{n}\left(t, y^{\prime}, z^{\prime}\right)\right| & \leq \frac{t^{2} e^{-n t}}{2(2 \pi-t)^{2}(t+n)^{2}} \sum_{i=n}^{\infty} \frac{1}{(i+t)^{2}}\left(\left|y_{i}^{2}-y_{i}^{\prime 2}\right|+\left|z_{i}^{2}-z_{i}^{\prime 2}\right|\right) \\
& \leq \frac{1}{2(2 \pi-1)^{2}} \sum_{i=1}^{\infty} \frac{1}{i^{2}}\left(\left|y+y^{\prime}\right|\left|y-y^{\prime}\right|+\left|z+z^{\prime}\right|\left|z-z^{\prime}\right|\right) \\
& \leq L\left(\left|y-y^{\prime}\right|+\left|z-z^{\prime}\right|\right)
\end{aligned}
$$

where $L=\frac{\pi^{2} r}{6(2 \pi-1)^{2}}$ and $r$ is the radius of the closed ball $\mathscr{B}$ which is fully compatible with the assumption $\left(\mathcal{H}_{1}\right)$. It is easy to see that

$$
\begin{aligned}
\left|f_{n}(t, y, z)\right| & \leq \frac{e^{-n t} \cos ^{2}(n t)}{(t+1)^{2}}+\frac{t^{2} e^{-n t}}{2(2 \pi-t)^{2}(t+n)^{2}} \sum_{i=n}^{\infty} \frac{1}{(i+t)^{2}}\left(\left|y_{n}\right|^{2}+\left|z_{n}\right|^{2}\right) \\
& =g_{n}(t)+h_{n}(t)\left(\left|y_{n}\right|^{2}+\left|z_{n}\right|^{2}\right)
\end{aligned}
$$

which is fully coincident with the assumption $\left(\mathcal{H}_{2}\right)$, where

$$
g_{n}(t)=\frac{e^{-n t} \cos ^{2}(n t)}{(t+1)^{2}}, \quad h_{n}(t)=\frac{t^{2} e^{-n t}}{2(2 \pi-t)^{2}(t+n)^{2}} \sum_{i=n}^{\infty} \frac{1}{(i+t)^{2}} .
$$

According the third assumption $\left(\mathcal{H}_{3}\right)$, we find that 


$$
\mathrm{C}=\sup _{n \in \mathbb{N}} \max _{t \in[0,1]}\left|h_{n}(t)\right|^{q-1}=\left(\frac{\pi^{2}}{12(2 \pi-1)^{2}}\right)^{\frac{1}{2}}=\frac{\pi}{\sqrt{12}(2 \pi-1)} \simeq 0.171658 .
$$

Therefore,

$$
\Delta=4^{(q-2) H(q-2)} \mathrm{C}\left(\Delta_{v}+\Delta_{v-\alpha}\right) \simeq(0.5)(0.171658)(1.90248+1.78358)=0.316371<1
$$

These lead to all assumptions of the theorem are satisfied. Hence, the infinite system (1) and (2) has at least one solution in $[0,1]$.

\section{Conclusions}

In the present study, we investigated an infinite system of fractional order with $p$ Laplacian operator. We used the Caputo-Katugampola derivative in our model, which related to several well-known fractional derivatives. The existence of solution to our infinite system is discussed by using the Darbo's fixed point theorem through applying the Hausdorff measure of noncompactness technique. A new sequence space related to $c_{0}$ space is presented to be our domain. An illustrated numerical example is provided to show that the applicability of our idea in practice.

Author Contributions: A.S., L.A. and F.A. contributed equally and significantly in writing this paper. All authors have read and agreed to the published version of the manuscript.

Funding: This project was funded by the Deanship of Scientific Research (DSR), King Abdulaziz University, Jeddah, grant number (G:001-130-1442). The authors, therefore, gratefully acknowledge DSR technical and financial support.

Informed Consent Statement: Not applicable

Data Availability Statement: Not applicable.

Acknowledgments: We are very grateful to the anonymous referees for their suggestions and valuable advices.

Conflicts of Interest: The authors declare that they have no conflicts of interest.

\section{References}

1. Jong, K.; Choi, H.; Ri, Y. Existence of positive solutions of a class of multi-point boundary value problems for $p$-Laplacian fractional differential equations with singular source terms. Commun. Nonlinear Sci. Numer. Simulat. 2019, 72, 272-281. [CrossRef]

2. Wang, P.; Ru, Y. Some existence results of positive solutions for $p$-Laplacian systems. Bound. Value Probl. 2019, 2019, 9. [CrossRef]

3. Pang, H.; Ge, W.; Tian, M. Solvability of nonlocal boundary value problems for ordinary differential equation of higher order with a $p$-Laplacian. Comput. Math. Appl. 2008, 56, 127-142. [CrossRef]

4. Salem, A.; Alshehri, H.M.; Almaghamsi, L. Measure of noncompactness for an infinite system of fractional Langevin equation in a sequence space. Adv. Differ. Equ. 2021, 2021, 132. [CrossRef]

5. Salem, A.; Alzahrani, F.; Alnegga, M. Coupled system of non-linear fractional Langevin equations with multi-point and nonlocal integral boundary conditions. Math. Probl. Eng. 2020, 2020, 7345658. [CrossRef]

6. Seemab, A.; Rehman, M. Existence of solution of an infinite system of generalized fractional differential equations by Darbo's fixed point theorem. J. Comput. Appl. Math. 2020, 364, 112355. [CrossRef]

7. Saadati, R.; Pourhadi, E.; Mursaleen, M. Solvability of infinite systems of third-order differential equations in $c_{0}$ by Meir-Keeler condensing operators. J. Fixed Point Theory Appl. 2019, 21, 1-16. [CrossRef]

8. Mursaleen, M.; Bilalov, B.; Rizvi, S.M.H. Applications of Measures of Noncompactness to Infinite System of Fractional Differential Equations. Filomat 2017, 31, 3421-3432. [CrossRef]

9. Mainradi, F.; Pironi, P. The fractional Langevin equation: Brownian motion revisited. Extr. Math. 1996, 10, $140-154$.

10. Sun, H.-G.; Chen, Y.-Q.; Chen, W. Random order fractional differential equation models. Signal Process. 2011, 91, 525-530. [CrossRef]

11. Salem, A. Existence results of solutions for anti-periodic fractional Langevin equation. J. Appl. Anal. Comput. 2020, 10, $2557-2574$. [CrossRef]

12. Salem, A.; Mshary, N. On the Existence and Uniqueness of Solution to Fractional-Order Langevin Equation. Adv. Math. Phys. 2020, 2020, 8890575. [CrossRef]

13. Salem, A.; Alzahrani, F.; Almaghamsi, L. Fractional Langevin equations with nonlocal integral boundary conditions. Mathematics 2019, 7, 402. [CrossRef] 
14. Salem, A.; Alzahrani, F.; Alghamdi, B. Langevin equation involving two fractional orders with three-point boundary conditions. Differ. Integral Equ. 2020, 33, 163-180.

15. Zhou, H.; Alzabut, J.; Yang, L. On fractional Langevin differential equations with anti-periodic boundary conditions. Eur. Phys. J. Spec. Top. 2017, 226, 3577-3590. [CrossRef]

16. Katugampola, U.N. Existence and uniqueness results for a class of generalized fractional differential equations. arXiv 2016, arXiv:1411.5229.

17. Almeida, R.; Malinowska, A.B.; Odzijewicz, T. Fractional Differential Equations With Dependence on the Caputo-Katugampola Derivative. J. Comput. Nonlinear Dyn. 2016, 11, 061017. [CrossRef]

18. Zeng, S.; Baleanu, D.; Bai, Y.; Wu, G. Fractional differential equations of Caputo-Katugampola type and numerical solutions. Appl. Math. Comput. 2017, 315, 549-554. [CrossRef]

19. Sweilam, N.H.; Nagy, A.M.; Al-Ajami, T.M. Numerical solutions of fractional optimal control with Caputo-Katugampola derivative. Adv. Differ. Equ. 2021, 2021, 425. [CrossRef]

20. Ho, V.; Ngo, V.H. Non-instantaneous impulses interval-valued fractional differential equations with Caputo-Katugampola fractional derivative concept. Fuzzy Sets Syst. 2021, 40, 111-140. [CrossRef]

21. Sun, H.; Chen, W.; Chen, Y. Variable-order fractional differential operators in anomalous diffusion modeling. Phys. A Stat. Mech. Its Appl. 2009, 388, 4586-4592. [CrossRef]

22. Wang, Y.; Gu, L.; Xu, Y.; Cao, X. Practical tracking control of robot manipulators with continuous fractional-order nonsingular terminal sliding mode. IEEE Trans. Ind. Electron. 2016, 63, 6194-6204. [CrossRef]

23. Machado, J.; Mata, M.E. A fractional perspective to the bond graph modeling of world economies. Nonlinear Dyn. 2015, 80, 1839-1852. [CrossRef]

24. Salem, A.; Alghamdi, B. Multi-Strip and Multi-Point Boundary Conditions for Fractional Langevin Equation. Fractal Fract. 2020, 42, 18. [CrossRef]

25. Salem, A.; Alghamdi, B. Multi-point and anti-periodic conditions for generalized Langevin equation with two fractional orders. Fractal Fract. 2019, 3, 51. [CrossRef]

26. Baghani, H. An analytical improvement of a study of nonlinear Langevin equation involving two fractional orders in different intervals. J. Fixed Point Theory Appl. 2019, 21, 95. [CrossRef]

27. Salem, A.; Al-dosari, A. Positive Solvability for Conjugate Fractional Differential Inclusion of $(k, n-k)$ Type without Continuity and Compactness. Axioms 2021, 10, 170. [CrossRef]

28. Salem, A.; Alnegga, M. Measure of Noncompactness for Hybrid Langevin Fractional Differential Equations. Axioms 2020,9 , 59. [CrossRef]

29. Banas, J.; Goebel, K. Measures of Noncompactness in Banach Spaces. In Lecture Notes in Pure and Applied Mathematics; Dekker: New York, NY, USA, 1980; Volume 60.

30. Katugampola, U.N. New approach to a generalized fractional integral. Appl. Math. Comput. 2011, 218, 860-865. [CrossRef]

31. Jarada, F.; Abdeljawadb, T.; Baleanu, D. On the generalized fractional derivatives and their Caputo modification. J. Nonlinear Sci. Appl. 2017, 10, 2607-2619. [CrossRef]

32. Katugampola, U.N. A new approach to generalized fractional derivatives. Bull. Math. Anal. Appl. 2014, 6, 1-15.

33. Liang, Z.; Han, X.; Li, A. Some properties and applications related to the (2, p)-Laplacian operator. Bound. Value Probl. 2016, 2016, 58. [CrossRef]

34. Damascelli, L. Comparison theorems for some quasilinear degenerate elliptic operators and applications to symmetry and monotonicity results. Ann. Inst. Henri Poincare Anal. Non Lineaire 1998, 15, 493-516. [CrossRef]

35. Banas, J.; Krajewska, M. Existence of solutions for infinite systems of differential equations in spaces of tempered sequences. Electron. J. Differ. Equ. 2017, 60, 1-28.

36. Banas, J.; Mursaleen, M. Sequence Spaces and Measures of Noncompactness with Applications to Differential and Integral Equations; Springer: New Delhi, India, 2014. 